\title{
Análisis de las citas de las publicaciones seriadas referenciadas en la revista Biomédica del Instituto Nacional de Salud Número 1, volumen 15, 1995, a número 2, volumen 20, 2000
}

\author{
Hernán Darío Higuita \\ Facultad Nacional de Salud Pública, Universidad de Antioquia, Medellín, Colombia.
}

\begin{abstract}
El propósito de este estudio es identificar y evaluar las referencias citadas en la revista Biomédica del Instituto Nacional de Salud. Se analizaron los siguientes aspectos: publicaciones citadas, cantidad de referencias, idioma, procedencia de las publicaciones citadas, obsolescencia, décadas citadas, producción y afiliación de los autores. A partir del análisis de esta información, se han corroborado comportamientos típicos en el terreno de la publicación de trabajos científicos, como son la escasa presencia de referencias de Latinoamérica frente a los países desarrollados, la concentración de referencias en un reducido número de títulos, el predominio del idioma inglés, la concentración de artículos en un reducido número de autores y la utilización de referencias de mayor actualidad.
\end{abstract}

Palabras clave: bibliometría, Biomédica, análisis de citas.

Quotation analysis of reference serial publications in Biomédica, journal of the Instituto Nacional de Salud, No.1, volume 15, 1995, to No.2, volume 20, 2000

The purpose of this study is to identify and evaluate the quoted references of Biomédica, journal of the Instituto Nacional de Salud. The following aspects were analyzed: quoted publications, number of references, language, origin of the quoted publications, obsolescence, quoted decades, production and affiliation of the authors. Typical characteristics pertaining to the domain of scientific publications were confirmed, based on the analysis of information collected. Among these characteristics, the following should be pointed out: a scarce presence of Latin American references in sharp contrast with those from developed countries, a concentration of references in a reduced number of titles, predominance of the English language, concentration of the articles in a reduced number of authors, and an extensive use of updated references.

Key words: bibliometry, Biomédica, citation analysis.

Las publicaciones seriadas, por su periodicidad y actualidad, constituyen uno de los canales más adecuados para divulgar, dentro de la comunidad científica, los avances y resultados de la actividad investigativa (1). En el campo de la biomedicina, es especialmente importante este tipo de publicaciones dentro del conjunto del material citado en los informes y artículos producidos por los investigadores.

El presente trabajo hace una recopilación y análisis de las citas bibliográficas que corresponden a las

Correspondencia:

hhiguita@yahoo.com

Recibido: 19/12/00; aceptado: 04/05/01 publicaciones seriadas que fueron citadas en los artículos de la revista Biomédica del Instituto Nacional de Salud, durante el periodo comprendido entre el número 1 del volumen 15 de 1995 y el número 2 del volumen 20 del 2000.

La revista Biomédica es una publicación trimestral eminentemente científica. Forma parte del índice Nacional de Publicaciones Seriadas Científicas y Tecnológicas Colombianas de Colciencias y aparece reseñada en el índice de la Literatura Latinoamericana en Ciencias de la Salud (LILACS), en el Sistema de Información Bibliográfica Regional Andina (SIBRA) y en CAB Abstracts, Review of Medical and Veterinary Entomology. 
El análisis de citas se ha convertido en una herramienta importante y controvertida para determinar el impacto que una publicación científica o técnica tiene en el campo de su especialidad o temática (2). Por otra parte, el acto de citación incluye variantes que merecen un análisis de tipo sociológico (5) y, aunque sus resultados no determinan la calidad de una publicación, sí aportan elementos de análisis para un comité editorial o para la evaluación que una biblioteca o centro de documentación haga de su colección de publicaciones seriadas $(3,4)$.

\section{Metodología}

Se elaboró una base de datos en el programa Access de Microsoft 1997. Se diseñaron dos tablas: una, con la información de las referencias bibliográficas contenidas en cada artículo, en las que se hubiese citado una publicación seriada, y otra, con la información de los autores. Posteriormente, se analizaron, por medio de cuadros, los resultados obtenidos con el cruce de la información recopilada.

Para el propósito de este estudio, se seleccionaron todos los artículos que tuvieran referencias bibliográficas, sin importar si se trataba de editoriales, artículos originales, informes, revisión de literatura, cartas, etc.

\section{Resultados}

\section{Publicaciones seriadas, las más citadas}

Del total de 177 artículos, 117 (66,1\%) tienen un rango de 1 a 20 referencias. Un porcentaje muy similar se encontró en otros análisis de citas efectuados a otras publicaciones biomédicas. Aunque, en ocasiones, se podría considerar este rango como un número bajo de referencias, también es indicativo de una mayor responsabilidad de los autores por citar la bibliografía que realmente se consultó y que sirven de respaldo para sus trabajos.

Del total de 4.334 referencias revisadas en los 177 artículos, 3.294 (76\%) correspondió a publicaciones seriadas, principalmente revistas, lo cual confirma la importancia que tiene este tipo de publicaciones en la investigación biomédica.

\section{Idioma}

El inglés es el idioma predominante en las referencias bibliográficas citada por los autores, con 2.843 referencias $(86,3 \%)$. Este idioma es el más utilizado para el intercambio de la divulgación científica y, por ende, de la medicina. Influye igualmente el hecho de que las publicaciones de mayor impacto en la medicina proceden de Estados Unidos e Inglaterra (6). El idioma que le sigue es el español con 409 referencias (12,41\%).

\section{Procedencia de las publicaciones citadas y autocitas}

Las publicaciones seriadas de Estados Unidos son las más citadas, con 1.728 referencias $(52,46 \%)$, seguidas por las de Inglaterra, con 519 referencias $(15,76 \%)$. Las publicaciones colombianas aparecen en tercer lugar con 288 referencias $(8,74 \%)$, en lo que se destaca la citación que se hace de la revista Biomédica (cuadro 1).

De las 224 referencias que correspondían a autocitas, el 45,54\% corresponde a publicaciones colombianas. Se destaca cómo el $54,46 \%$ restante corresponde a artículos publicados en revistas internacionales.

\section{Distribución por zonas}

De los 174 artículos analizados, se obtuvieron 3.294 referencias que corresponden a 795 títulos de publicaciones seriadas. Después de organizar esos títulos en orden descendente de acuerdo con la cantidad de referencias citadas, se procedió a dividirlos en tres zonas, cada una de las cuales

Cuadro 1. Procedencia de las publicaciones seriadas citadas.

\begin{tabular}{lcc}
\hline País & Referencias & Porcentaje \\
\hline Estados Unidos & 1.728 & 52,46 \\
Inglaterra & 519 & 15,76 \\
Colombia & 288 & 8,74 \\
Sin datos & 132 & 4,00 \\
Holanda & 107 & 3,25 \\
Suiza & 103 & 3,13 \\
Brasil & 83 & 2,52 \\
Otros países & 334 & 10,14 \\
Total & $\mathbf{3 . 2 9 4}$ & $\mathbf{1 0 0 , 0 0}$ \\
\hline
\end{tabular}


contenía un tercio del total de las citas, es decir, 1.098 cada una (cuadro 2).

La primera zona comprende 21 publicaciones, de lo que se desprende que una tercera parte del total de las citas referenciadas por los autores de Biomédica (1.097) están en sólo 21 publicaciones.

La segunda zona contiene 104 títulos, que representan el segundo tercio del total de citas.

La tercera zona contiene 670 publicaciones, que constituyen el último tercio. De este último grupo, se encuentran 426 publicaciones que sólo fueron citadas una sola vez en el período estudiado de Biomédica y 126 fueron citadas solamente dos veces.

\section{Revistas más citadas}

Es importante destacar que Biomédica es la segunda revista más citada dentro del conjunto de publicaciones seriadas, después del American Journal of Tropical Medicine and Hygiene (cuadro 3). Esto obedece a que la mayoría de los autores están vinculados con el Instituto Nacional de Salud y a la importancia que tiene esta publicación dentro

Cuadro 2. Distribución de títulos por zonas.

\begin{tabular}{lcc}
\hline Zona & Títulos & Referencias \\
\hline Primera & 21 & 1.097 \\
Segunda & 104 & 1.098 \\
Tercera & 670 & 1.099 \\
Total & 795 & $\mathbf{3 . 2 9 4}$ \\
\hline
\end{tabular}

del conjunto de las publicaciones seriadas en el área de la salud en el país.

\section{Obsolescencia}

De acuerdo con la distancia en años entre la fecha en que un artículo fue publicado y la fecha de sus referencias bibliográficas, se observa que el mayor grupo de ellas se encuentra en un nivel de obsolescencia de 0 a 10 años (cuadro 4). Este rango concuerda con otros trabajos que se han hecho en publicaciones biomédicas y ratifica la importancia que tiene la información actualizada para los investigadores en las áreas de la salud $(1,3)$.

La década más citada en las referencias compiladas corresponde al periodo 1991-2000, con 1.571 referencias $(47,69 \%)$. Ello indica que, cuantitativamente, esta década produjo la información más importante para los autores de los artículos. Le sigue la década 1981-1990, con 1.065 referencias $(32,33 \%)$.

\section{Producción de los autores}

Del total de autores, individualmente considerados, un alto porcentaje, 350 autores $(89,52 \%)$, sólo figuran entre una y dos veces. Si se toma este dato aisladamente - sin apelar a otras variables -, se podría concluir que un altísimo porcentaje de autores de la revista investiga de manera individual. Aunque sabemos que, en realidad, los trabajos

Cuadro 3. Títulos de revistas más citadas.

Título de la publicación

\begin{tabular}{cc}
$\begin{array}{c}\text { Número de referencias } \\
\text { de un total de } \mathbf{3 . 2 9 4}\end{array}$ & Porcentaje \\
\hline 116 & 3,52 \\
102 & 3,10 \\
70 & 2,13 \\
63 & 1,91 \\
62 & 1,88 \\
60 & 1,82 \\
59 & 1,79 \\
58 & 1,76 \\
55 & 1,67 \\
52 & 1,58 \\
46 & 1,40 \\
43 & 1,31 \\
41 & 1,24 \\
41 & 1,24 \\
38 & 1,15 \\
\hline
\end{tabular}


Cuadro 4. Obsolescencia de las referencias citadas.

\begin{tabular}{lcr}
\hline $\begin{array}{l}\text { Período } \\
\text { (años) }\end{array}$ & $\begin{array}{c}\text { Referencias } \\
\text { (número) }\end{array}$ & Porcentaje \\
\hline 0 a 10 & 2.136 & 64,85 \\
11 a 20 & 682 & 20,70 \\
21 a 30 & 267 & 8,11 \\
31 o más & 203 & 6,16 \\
Sin datos & 6 & 0,18 \\
Total & $\mathbf{3 . 2 9 4}$ & $\mathbf{1 0 0 , 0 0}$ \\
\hline
\end{tabular}

de investigación requieren durante su proceso del concurso de profesionales de diferentes disciplinas, es importante destacar cómo el resultado de esos procesos, expresado en un artículo para una revista, se hace de manera individual.

Es normal encontrar grupos de autores que son constantes en la producción de artículos; es así como, en Biomédica, el $10,48 \%$ de los autores que figuran tres o más veces produjeron el $35,37 \%$ del total de todos los artículos en el período estudiado (cuadro 5). Existe la tendencia a que mientras más trabajos haya producido un autor, más facilidad tiene para elaborar otros.

\section{Número de autores por artículo}

El $62,14 \%$ de los artículos figuran con tres o más autores. Hay que tener en cuenta que los trabajos de investigación médica requieren, por su complejidad, de la participación de varios profesionales, provenientes muchas veces de diferentes disciplinas o áreas. Los artículos que se publican dan cuenta de este trabajo colectivo.

\section{Afiliación de los autores}

La revista Biomédica está vinculada con el Instituto Nacional de Salud; por ello, el mayor porcentaje de los autores de los artículos se encuentra afiliados a él. Sin embargo, es importante destacar la relación que esta publicación tiene con los principales centros de educación superior del país, como son la Universidad Nacional, la Universidad de Cartagena, la Universidad de Antioquia y la Universidad Industrial de Santander (cuadro 6).

\section{Conclusión}

El análisis de citas de la revista Biomédica corrobora el comportamiento típico en la producción de artículos en revistas científicas y técnicas en países en vías de desarrollo, tal como se ha señalado en otros estudios afines $(3,4)$. Entre las constantes que se verifican, se cuentan: poca presencia de publicaciones latinoamericanas; fuerte impacto de las publicaciones de países desarrollados; el inglés como idioma principal; la concentración de referencias en un grupo reducido de títulos, y un mayor porcentaje de referencias en un nivel de obsolescencia de 0 a 10 años, entre otras.

Cuadro 5. Producción de los autores.

\begin{tabular}{|c|c|c|c|c|}
\hline \multirow[t]{2}{*}{$\begin{array}{l}\text { Número de veces } \\
\text { que aparece }\end{array}$} & \multicolumn{2}{|c|}{$\begin{array}{l}\text { Autores individualmente } \\
\text { considerados }\end{array}$} & \multicolumn{2}{|c|}{ Agrupados por autoría } \\
\hline & Cantidad & Porcentaje & Cantidad & Porcentaje \\
\hline 1 & 300 & 76,73 & 300 & 48,47 \\
\hline 2 & 50 & 12,79 & 100 & 16,16 \\
\hline 3 & 14 & 3,58 & 42 & 6,79 \\
\hline 4 & 7 & 1,78 & 28 & 4,52 \\
\hline 5 & 7 & 1,78 & 35 & 5,65 \\
\hline 6 & 4 & 1,02 & 24 & 3,88 \\
\hline 7 & 1 & 0,26 & 7 & 1,13 \\
\hline 8 & 1 & 0,26 & 8 & 1,29 \\
\hline 9 & 3 & 0,77 & 27 & 4,36 \\
\hline 10 & 2 & 0,51 & 20 & 3,23 \\
\hline 13 & 1 & 0,26 & 13 & 2,10 \\
\hline 15 & 1 & 0,26 & 15 & 2,42 \\
\hline Total & 391 & 100,00 & 619 & 100,00 \\
\hline
\end{tabular}


Cuadro 6. Afiliación de los autores.

\begin{tabular}{lcc}
\hline Institución & Autores & Porcentaje \\
\hline Instituto Nacional de Salud & 128 & 32,74 \\
Universidad Nacional de Colombia & 28 & 7,16 \\
Universidad de Cartagena & 20 & 5,12 \\
Universidad de Antioquia & 13 & 3,32 \\
Universidad Industrial de Santander & 10 & 2,56 \\
Corporación para Investigaciones Biológicas & 10 & 2,56 \\
Secretaría de Salud del Amazonas & 8 & 2,05 \\
Universidad de La Salle & 7 & 1,79 \\
Hospital Vozandes, Ecuador & 6 & 1,53 \\
Instituto Colombiano de Medicina Tropical & 6 & 1,53 \\
Universidad Pontificia Bolivariana & 6 & 1,53 \\
Pontificia Universidad Javeriana & 6 & 1,53 \\
Universidad Colegio Mayor de Cundinamarca & 6 & 1,53 \\
Universidad de los Andes & 6 & 1,53 \\
Universidad del Valle & 6 & 1,53 \\
Universidad del Quindío & 5 & 1,28 \\
National Institute of Health, Bethesda, USA & 5 & 1,28 \\
Centro Internacional de Restauración Neurológica & 5 & 1,28 \\
Instituto Nacional de Salud y Universidad Nacional & 4 & 1,02 \\
Otras instituciones & 106 & 27,13 \\
Total & 391 & 100,00 \\
\hline
\end{tabular}

No se pueden desconocer las dificultades que debe afrontar una publicación de su tipo en el contexto de un país como Colombia, donde, además de lidiar con las dificultades económicas, los investigadores - por su parte - se encuentran con el afán de divulgar sus artículos en publicaciones que gozan de mayor prestigio en el ámbito internacional y que, por múltiples circunstancias, se encuentran en los países desarrollados. Se genera, de esta manera, un círculo vicioso en el que las publicaciones locales no alcanzan a cumplir con los parámetros internacionales por no publicar en ellas autores de prestigio, pero, a su vez, éstos no publican en las revistas locales por carecer éstas de dicho prestigio internacional.

Existe la necesidad de generar en nuestros países espacios de investigación y reflexión que indaguen las circunstancias reales en lo concerniente a la producción científica y a la divulgación de sus desarrollos en las producciones seriadas.

\section{Referencias}

1. Cañedo R. Los análisis de citas en la evaluación de los trabajos científicos y las publicaciones seriadas. ACIMED 1999;7:30-9.

2. Garfield E. Análisis cuantitativo de la literatura científica y sus repercusiones en la formulación de políticas científicas en América Latina y el Caribe. Bol Oficina Sanit Panam 1995;118:448-56.

3. Higuita HD. Estudio bibliométrico de la revista latreia de la Facultad de Medicina de la Universidad de Antioquia, 1988-1997 (tesis). Medellín: Universidad de Antioquia; 1998.

4. Sancho R. Indicadores bibliométricos utilizados en la evaluación de la ciencia y la tecnología. Rev Española Docum Cient 1990;13:842-65.

5. Spinak E. Los análisis cuantitativos de la literatura científica y su validez para juzgar la producción latinoamericana. Bol Oficina Sanit Panam1996; 120:139-47.

6. Ulrich's International Periodicals Directory: a classified guide to current periodicals foreign and domestic. Estados Unidos: Bowker; 1996. 